\title{
Refractory anaplastic astrocytoma responsive to PCV in combination with bevacizumab
}

\author{
Arun Swaminathan ${ }^{1}$, Donita D. Lightner ${ }^{1}$, Thomas Pittman ${ }^{2}$, Craig Horbinski ${ }^{3}$, John Lee Villano ${ }^{1,4}$ \\ 1. Departments of Neurology, University of Kentucky Chandler Medical Center, Lexington, Kentucky, United States. \\ 2. Neurosurgery, University of Kentucky Chandler Medical Center, Lexington, Kentucky, United States. 3. Pathology, \\ University of Kentucky Chandler Medical Center, Lexington, Kentucky, United States. 4. Medicine, University of Kentucky \\ Chandler Medical Center, Lexington, Kentucky, United States.
}

Correspondence: Arun Swaminathan. Address: Department of Neurology, University of Kentucky, 740 S Limestone St, L 445, Lexington, KY, United States. E-mail: asw224@uky.edu

Received: September 5, 2014

Accepted: January 26, 2015

Online Published: March 11, 2015

DOI : $10.5430 /$ crcp.v2n3p17

URL: http://dx.doi.org/10.5430/crcp.v2n3p17

\begin{abstract}
We present a patient with anaplastic astrocytoma who had recurrent disease after treatment with surgery, radiation, procarbazine, lomustine (CCNU) and vincristine (PCV) over one year followed by poor response to second line treatment with temozolomide, irinotecan with bevacizumab, Novocure TTF therapy successively over a four year period after her initial treatment who then responded to PCV in combination with bevacizumab as third line therapy. This is the first report demonstrating benefit of concurrent PCV and bevacizumab treatment in a highly treatment refractory tumor.
\end{abstract}

\section{Key words}

Anaplastic astrocytoma, Brain tumor, Tumor treatment, Recurrent tumor

\section{I ntroduction}

Approximately $40 \%$ of primary brain tumors are gliomas and about $8.3 \%$ of these cases are anaplastic astrocytomas, making it difficult to perform large randomized clinical trials1. Unfortunately, these high-grade astrocytomas are also associated with a poor prognosis with a 5-year survival of approximately $28 \%{ }^{[1]}$. Recurrent tumors represent a treatment conundrum as they defy standard treatment and require second line therapy. Evidence from glioblastoma studies, professional and anecdotal experience act as determinants of choice of therapy due to a paucity of established guidelines. Targeted chemotherapy with vascular endothelial growth factor (VEGF) inhibitors, like bevacizumab, in conjunction with conventional chemotherapy agents, such as irinotecan or CCNU, offers more treatment options for progressive disease. Bevacizumab has previously been used concomitantly with temozolomide, irinotecan, carboplatin, and CCNU. We present an unusual case of a patient with recurrent anaplastic astrocytoma who had disease recurrence with first- and second-line chemotherapy, repeated surgical resections, Novocure TTF therapy and repeated radiation therapy who finally responded to PCV with bevacizumab. Trials with other targeted agents are ongoing but formal guidelines are yet to be established. Therapy for recurrent tumors is individualized according to patient's status, prior treatment, side effect profile and the treating physician's personal expertise. Genetic testing for molecular markers and targeted therapies to enable better prognostication and therapeutic efficacy is ongoing and continues to be a major source of research in this field. 


\section{History and presentation}

A 31-year-old female presented in March 2002 with recurrent, severe unilateral headaches in the left frontal region. Magnetic resonance imaging (MRI) demonstrated a left frontal tumor (see Figure 1). Upon further history, she had a sister with oligodendroglioma and her mother had a history of brain tumor, pathology unknown. Gross total resection was performed and histopathology showed a low grade oligodendroglioma. After surgery, the patient received chemotherapy with six cycles of PCV from May 2002 through March 2003. She recovered and had minimal residual deficits on follow up.

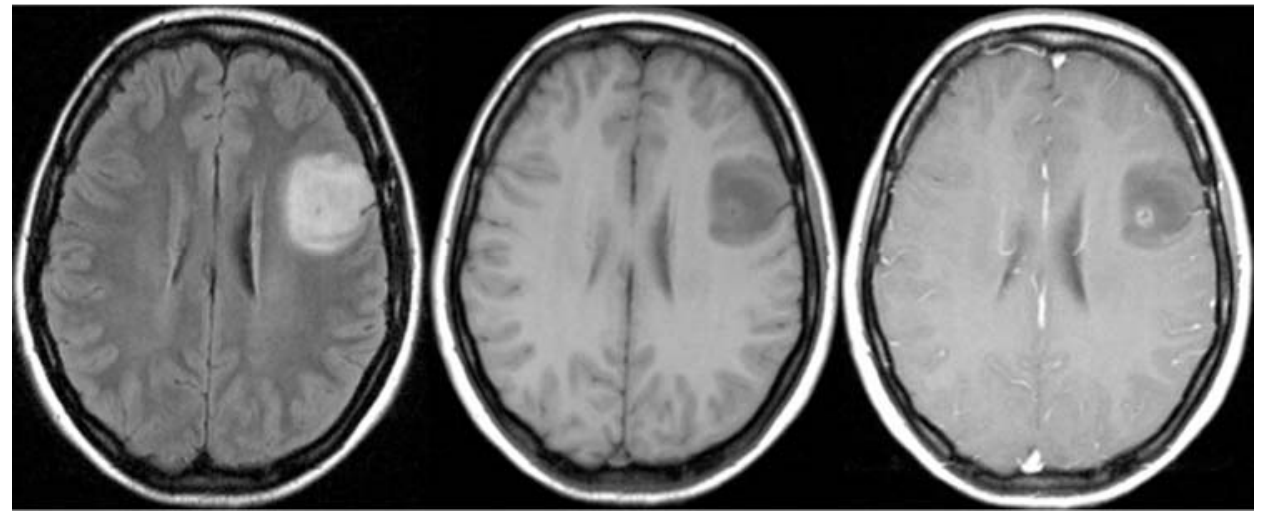

Figure 1. Fluid attenuated inversion recovery (FLAIR) sequence, T1 weighted imaging without contrast, and T1 weighted imaging with contrast axial cuts of initial imaging

She returned in February 2008 with severe headaches when a repeat MRI showed recurrence of disease at the same location (see Figure 2). A repeat resection with implantation of carmustine wafers (polifeprosan 20 with carmustine implant [Gliadel ${ }^{\circledR}$ wafer], MGI Pharma Inc.) was performed. Pathology suggested a recurrent anaplastic high grade oligodendroglioma. Chromosome 1p/19q co-deletion testing was not performed. She was treated with twelve cycles of temozolomide at $150 \mathrm{mg} / \mathrm{m}^{2}$ for 5 days at 28 day intervals, being on it for 3 weeks and off it for 1 week, from July 2008 2009 and remained asymptomatic during this time.

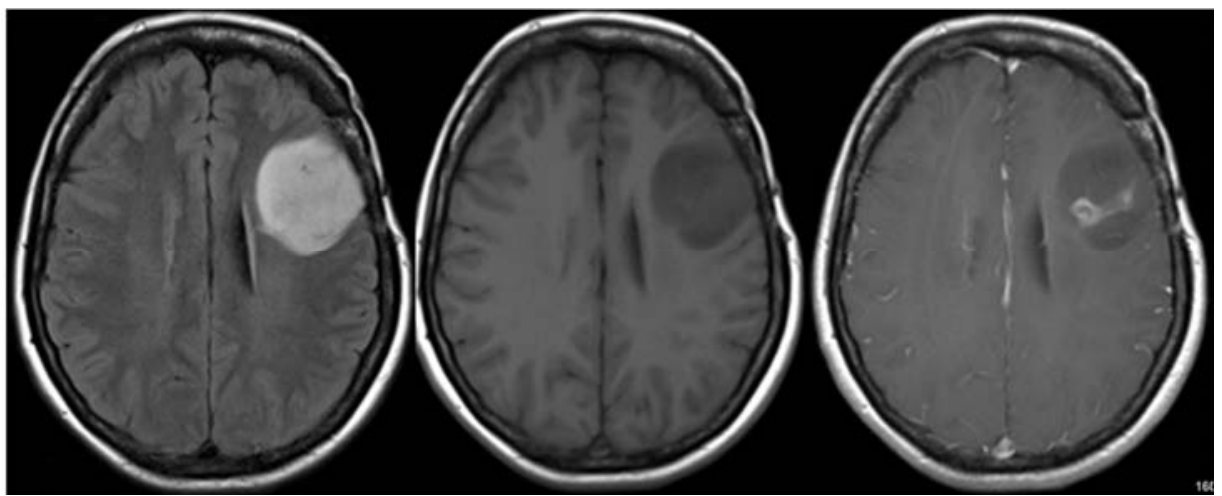

Figure 2. FLAIR, T1 weighted imaging without contrast, and T1 weighted imaging with contrast at the time of recurrence in 2008

She returned with seizures in June 2009 and MRI showed progression of the tumor around the left lateral ventricle and margin of left optic cavity (see Figure 3). She was advised repeat resection and whole brain irradiation but she declined the surgery, and opted for chemotherapy with radiation. She then received temozolomide therapy daily at $75 \mathrm{mg} / \mathrm{m}^{2}$ for three weeks with alternate weekly therapy, along with simultaneous whole brain irradiation therapy, followed by adjuvant temozolomide therapy at $150 \mathrm{mg} / \mathrm{m}^{2}$ for five days given at 28 day intervals for another year. This was followed by irinotecan and bevacizumab over the next seven months. 
Figure 3. Axial FLAIR (top) and T1-weighted imaging with contrast (bottom) at time of recurrence in June 2009

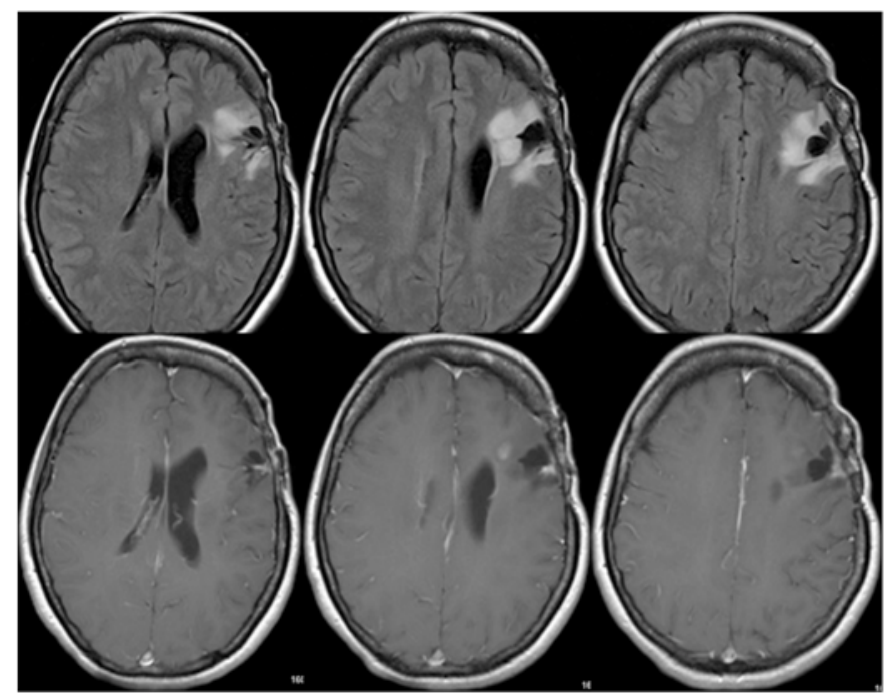

She had a partial surgical resection in August 2012 which was initially diagnosed as a recurrent left frontal anaplastic oligodendroglioma. However, because there was some question as to whether this tumor was better classified as an astrocytoma, her 2008 case was retrieved from the archives for additional molecular testing. Fluorescence in situ hybridization (FISH) analysis of her 2008 tumor showed no 1p/19q codeletion (not shown). Furthermore, while her tumor was immunopositive for R132H IDH1, it showed loss of nuclear alpha-thalassemia/mental retardation syndrome X-linked (ATRX) expression (see Figure 4). Thus, the tumor was reclassified as an anaplastic astrocytoma.
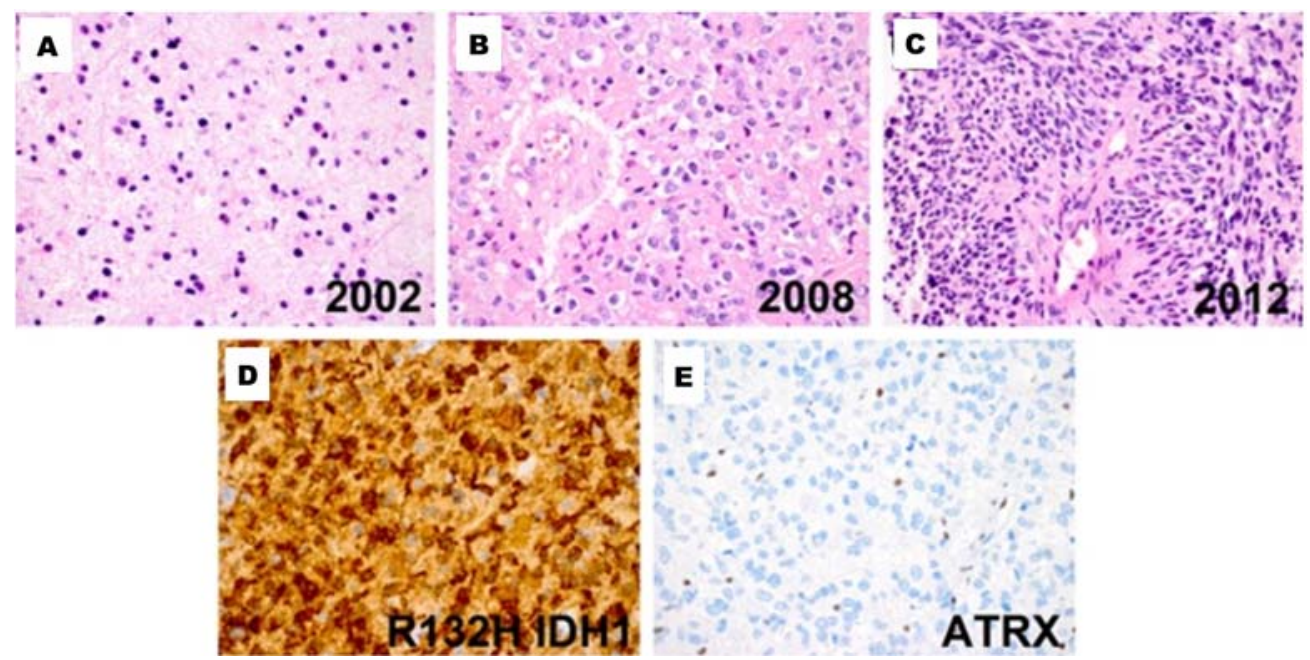

ATRX

Figure 4. Neuro-pathothology slides from the initial resection (A) and then in 2009 (B) and 2012 (C). Slide (D) indicates the R132 IDH1 positivity and (E) showing nuclear loss of ATRX.

As a break from systemic chemotherapy, Novocure TTF-100A device therapy was considered for the residual tumor. She however, discontinued the device within one week as she could not tolerate wearing the device due to claustrophobia. Her compliance was consequently less than $10 \%$, with the goal of $75 \%$ of time on active therapy with the TTF-100A device.

She presented to the emergency room with seizures, new onset expressive aphasia, and her baseline right hemiparesis in January 2013. MRI showed recurrence of disease along the left frontal lobe towards the corpus callosum. She declined further surgery and instead opted for further radiation and chemotherapy. She was started on helical tomotherapy with re-start of bevacizumab. When we were reviewing the literature for treatment options, we found that patients who received combinations of alkylating chemotherapeutic agents with bevacizumab seemed to have longer progression-free survival than patients treated with the same agents alone. Our patient had been treated with PCV previously, and had demonstrated 
initial response to therapy. In conclusion, we felt using the three-drug combination with bevacizumab could confer greater benefit than the conventional chemotherapy alone, despite the potential risk of increased toxicity from multiple agents. In February 2013, our patient was started on monthly PCV with bevacizumab.

Our patient's seizures, aphasia, and even hemiparesis improved with therapy over the next few months using this atypical regimen. Repeat MRI scans in April 2013 showed response to treatment signified by replacement of tumor mass and edema with CSF signal (see Figure 5). She remained stable and demonstrated improved motor and speech functioning on this regimen for two months. Then, in June 2013, she had further clinical progression with worsening speech, facial tics, myoclonus, and dystonia. Later, she developed severe generalized weakness and failure to thrive necessitating nursing home admission for closer supervision. Her repeat MRI in October 2013 (approximately eight months after initiation) revealed worsening disease. It was at this time the family made the decision to pursue hospice and palliative care.

Figure 5. Panel A showing axial FLAIR (top) and T1-weighted imaging with contrast (bottom) demonstrating tumor progression in January 2013. While panel B shows the axial FLAIR (top) and T1-weighted imaging with contrast (bottom) indicating tumor response after PCV with bevacizumab in April 2013.

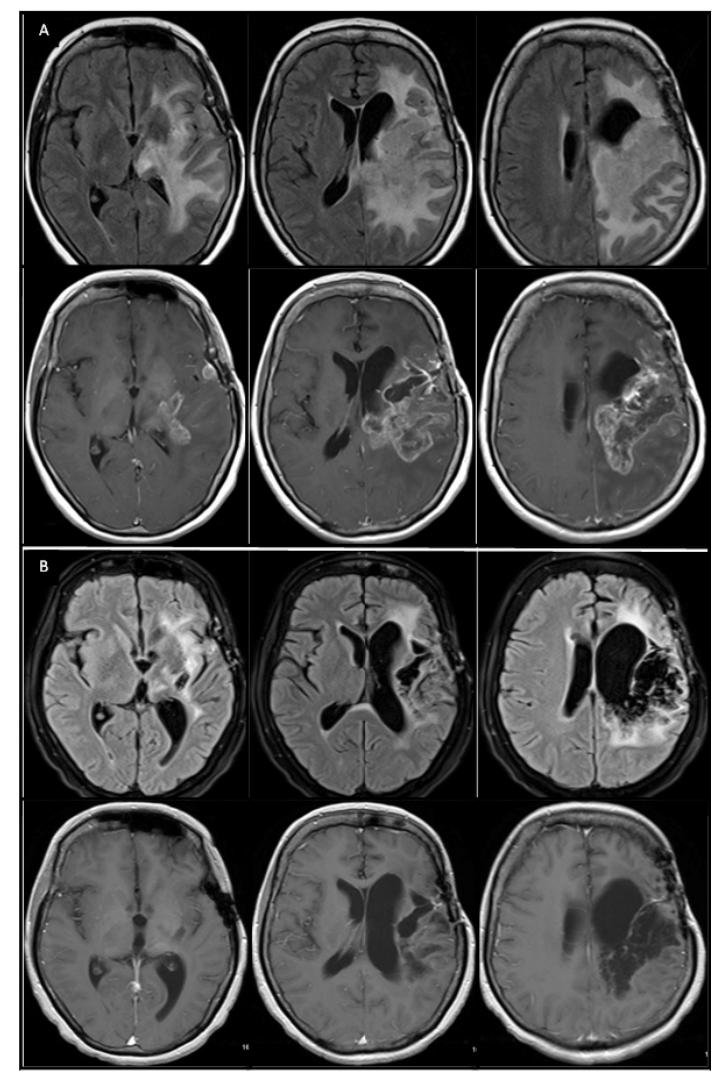

\section{Discussion}

Anaplastic astrocytomas are classified as grade III astrocytomas by the WHO classification of brain tumors and are high-grade tumors ${ }^{[2]}$. They are typically found in the frontal and parietal lobes with greatest predominance in the cerebral hemispheres and usually present with seizures or headaches. Signs of increased intracranial pressure such as rigidity, lethargy, and meningeal signs are less frequent ${ }^{[2]}$. MRI usually shows at least some contrast enhancement, indicating breakdown of the blood-brain barrier that is characteristic of high-grade gliomas.

This case illustrates the utility of molecular biomarkers in refining the classification of these tumors. They are an important component of therapeutic decision-making, diagnosis, and prognosis, as they have been shown to predict potential responses to treatment and make clinical trial assignment more accurate. The fact that this patient had an IDH1 mutation is not surprising, considering she was relatively young and had a protracted clinical course ${ }^{[3]}$. What was interesting was the 2008 tumor, despite still being histologically favored as having oligodendroglial lineage, lacked 1p/19q codeletion and 
instead had loss of nuclear ATRX expression (insufficient tissue was available from the 2002 tumor for testing). The ATRX gene product is a telomere binding protein that has loss of nuclear expression in $75 \%$ of grade II and III astrocytomas and secondary glioblastoma ${ }^{[4]}$. This loss activates the pathway of alternative lengthening of telomeres, a presumed precursor to genomic instability. This combination of intact 1p/19q and ATRX loss is, not only more consistent with an astrocytic lineage, but anaplastic gliomas with this pattern will behave like anaplastic astrocytomas regardless of their histologic appearance ${ }^{[4]}$. In light of that, and of the fact that the 2012 recurrence was far more morphologically consistent with an astrocytoma, the tumor was reclassified.

Treatment of these tumors involves the combination of surgical resection followed by radiation for maximum benefit. Maximal surgical resection has the greatest benefit as first-line therapy as compared to chemotherapy and radiation ${ }^{[5]}$.

Beyond initial surgical treatment, radiation and chemotherapy versus radiation alone can be debated. As anaplastic astrocytomas only represent a relatively small percentage of glioma patients, there are no prospective trials to date indicating benefit from early treatment; however, there is an ongoing phase III trial to investigate the benefit of the addition of temozolomide. Most providers are extrapolating from glioblastoma studies to treat these patients frontline. While a meta-analysis has suggested a survival benefit with front line chemoradiotherapy, there have been other studies which have failed to replicate the data ${ }^{[6-10]}$. There is category 1 evidence for newly diagnosed anaplastic astrocytoma supporting radiotherapy alone, given as 60 Grey in 30 fractions, and a single institutional retrospective review supports the evidence ${ }^{[11]}$. Our patient was treated originally based upon the initial histological diagnosis of oligodendroglioma and evidence from the phase III trial Radiation Therapy Oncology Group (RTOG) 9402 for anaplastic oligodendrogliomas and response to $\mathrm{PCV}{ }^{[12]}$.

Anaplastic astrocytomas frequently recur or undergo malignant degeneration to glioblastoma. These tumors, frequently, are unable to have repeated gross total surgical resection thereby making the role of chemotherapy and other modalities crucial for patients ${ }^{[13]}$. Debulking followed by chemotherapy, stereotactic radiosurgery, and adjuvant drugs in various combinations have been tried and no single combination has shown significantly greater efficacy than others. Chemotherapy agents used for recurrent anaplastic astrocystoma include temozolomide, nitrosoureas (carmustine, lomustine), procarbazine, irinotecan, cisretinoic acid, platinum compounds (cisplatin, carboplatin), and cyclophosphamide ${ }^{[13]}$. These agents have been tried in different combinations and have similar rates of response. However, in a trial comparing PCV vs temozolomide, the group treated with temozolomide suffered less toxicity and had less premature discontinuation of treatment ${ }^{[14]}$. As such, temozolomide is favored over PCV by some experts due to equal efficacy, fewer side effects, and ease of administration of a single agent over multiple agents ${ }^{[15]}$. Additionally, choice of chemotherapy agent for recurrent anaplastic astrocytoma depends on what prior treatment was used, whether PCV or temozolomide, or nitrosourea ${ }^{[16]}$. Carmustine polymer discs (i.e. gliadel wafer) implantation was shown to increase survival with minimal systemic toxicity ${ }^{[17]}$. NovoTTF-100A has demonstrated benefit for recurrent glioblastoma and, as such, would be a consideration for recurrent anaplastic astrocytoma ${ }^{[18,19]}$.

Targeted chemotherapy also involves the use of molecularly targeted compounds against VEGF to target tumor angiogenesis. Studies have demonstrated VEGF is up-regulated in high grade gliomas and the main mechanism of action involves normalization of abnormal tumor vasculature, thereby improving vasogenic edema. Other mechanisms may also include normalization of tumor blood flow and dose-delivery of chemotherapy to active tumor site. Theoretically it may increase efficacy of radiation by increasing oxygen delivery, thereby increasing oxidative damage. Bevacizumab has been used to treat temozolomide-refractory recurrent anaplastic astrocytomas with beneficial results ${ }^{[20]}$. It has also been used with cytotoxic agents like platinum compounds or irinotecan for anaplastic astrocytoma with encouraging results, all supporting its role for potential treatment as second-line chemotherapy for recurrent disease. There is evidence from glioblastoma studies supporting its use.

To our knowledge, there are no prior data or cases reporting response to PCV in combination with bevacizumab as therapy for highly refractory astrocytomas. We therefore hope that our case will expand treatment options for patients with recurrent disease. 


\section{References}

[1] See SJ, Gilbert MR. Anaplastic astrocytoma: diagnosis, prognosis, and management. Semin Oncol. 2004; 31(5): 618-34. http://dx.doi.org/10.1053/j.seminoncol.2004.07.004

[2] Omuro A, DeAngelis LM. Glioblastoma and other malignant gliomas: a clinical review. JAMA. 2013; 310(17): 1842-50. PMid: 24193082. http://dx.doi.org/10.1001/jama.2013.280319

[3] Horbinski C. What do we know about IDH1/2 mutations so far, and how do we use it? Acta Neuropathol. 2013; 125(5): 621-36. PMid: 23512379. http://dx.doi.org/10.1007/s00401-013-1106-9

[4] Wiestler B, Capper D, Holland-Letz T, et al. ATRX loss refines the classification of anaplastic gliomas and identifies a subgroup of IDH mutant astrocytic tumors with better prognosis. Acta Neuropathol. 2013; 126(3): 443-51. PMid: 2390411. http://dx.doi.org/10.1007/s00401-013-1156-z

[5] Hess KR. Extent of resection as a prognostic variable in the treatment of gliomas. J Neurooncol. 1999; 42(3): 227-31. PMid: 10433106. http://dx.doi.org/10.1023/A:1006118018770

[6] Stewart LA. Chemotherapy in adult high-grade glioma: a systematic review and meta-analysis of individual patient data from 12 randomised trials. Lancet. 2002; 359(9311): 1011-8. http://dx.doi.org/10.1016/S0140-6736(02)08091-1

[7] Kristiansen K, Hagen S, Kollevold T, et al. Combined modality therapy of operated astrocytomas grade III and IV. Confirmation of the value of postoperative irradiation and lack of potentiation of bleomycin on survival time: a prospective multicenter trial of the Scandinavian Glioblastoma Study Group. Cancer. 1981; 47(4): 649-52. http://dx.doi.org/10.1002/1097-0142(19810215)47:4<649::AID-CNCR2820470405>3.0.CO;2-W

[8] Randomized trial of procarbazine, lomustine, and vincristine in the adjuvant treatment of high-grade astrocytoma: a Medical Research Council trial. J Clin Oncol. 2001; 19(2): 509-18. PMid: 11208845.

[9] Hildebrand J, Gorlia T, Kros JM, et al. Adjuvant dibromodulcitol and BCNU chemotherapy in anaplastic astrocytoma: results of a randomised European Organisation for Research and Treatment of Cancer phase III study (EORTC study 26882). Eur J Cancer. 2008; 44(9): 1210-6. PMid: 18248979. http://dx.doi.org/10.1016/j.ejca.2007.12.005

[10] Wick W, Hartmann C, Engel C, et al. NOA-04 randomized phase III trial of sequential radiochemotherapy of anaplastic glioma with procarbazine, lomustine, and vincristine or temozolomide. J Clin Oncol. 2009; 27(35): 5874-80. PMid: 19901110. http://dx.doi.org/10.1200/JCO.2009.23.6497

[11] Shonka NA, Theeler B, Cahill D, et al. Outcomes for patients with anaplastic astrocytoma treated with chemoradiation, radiation therapy alone or radiation therapy followed by chemotherapy: a retrospective review within the era of temozolomide. J Neurooncol. 2013; 113(2): 305-11. PMid: 23526410. http://dx.doi.org/10.1007/s11060-013-1116-4

[12] Cairncross G, Wang M, Shaw E, et al. Phase III trial of chemoradiotherapy for anaplastic oligodendroglioma: long-term results of RTOG 9402. J Clin Oncol. 2013; 31(3): 337-43. PMid: 23071247. http://dx.doi.org/10.1200/JCO.2012.43.2674

[13] Chamberlain MC, Tsao-Wei DD, Groshen S. Salvage chemotherapy with cyclophosphamide for recurrent temozolomiderefractory anaplastic astrocytoma. Cancer. 2006; 106(1): 172-9. PMid: 16323194. http://dx.doi.org/10.1002/cncr.21582

[14] Brandes AA, Nicolardi L, Tosoni A, et al. Survival following adjuvant PCV or temozolomide for anaplastic astrocytoma. Neuro Oncol. 2006; 8(3): 253-60. PMid: 16723632. http://dx.doi.org/10.1215/15228517-2006-005

[15] Yung WK, Prados MD, Yaya-Tur R, et al. Multicenter phase II trial of temozolomide in patients with anaplastic astrocytoma or anaplastic oligoastrocytoma at first relapse. Temodal Brain Tumor Group. J Clin Oncol. 1999; 17(9): 2762-71. PMid: 10561351.

[16] Cairncross G, Berkey B, Shaw E, et al. Phase III trial of chemotherapy plus radiotherapy compared with radiotherapy alone for pure and mixed anaplastic oligodendroglioma: Intergroup Radiation Therapy Oncology Group Trial 9402. J Clin Oncol. 2006; 24(18): 2707-14. PMid: 16782910. http://dx.doi.org/10.1200/JCO.2005.04.3414

[17] Brem H, Piantadosi S, Burger PC, et al. Placebo-controlled trial of safety and efficacy of intraoperative controlled delivery by biodegradable polymers of chemotherapy for recurrent gliomas. The Polymer-brain Tumor Treatment Group. Lancet. 1995; 345(8956): 1008-12. http://dx.doi.org/10.1016/S0140-6736(95)90755-6

[18] Fonkem E, Wong ET. NovoTTF-100A: a new treatment modality for recurrent glioblastoma. Expert Rev Neurother. 2012; 12(8): 895-9. PMid: 22708931. http://dx.doi.org/10.1586/ern.12.80

[19] Davies AM, Weinberg U, Palti Y. Tumor treating fields: a new frontier in cancer therapy. Ann N Y Acad Sci. 2013; $1291:$ 86-95. PMid: 23659608. http://dx.doi.org/10.1111/nyas.12112

[20] Chamberlain MC, Johnston S. Salvage chemotherapy with bevacizumab for recurrent alkylator-refractory anaplastic astrocytoma. J Neurooncol. 2009; 91(3): 359-67. PMid: 18953491. http://dx.doi.org/10.1007/s11060-008-9722-2 